\title{
Reforming accelerated approval
}

\author{
Proposed US legislation aiming to expand and expedite patient access to novel drugs represents a good start, but is \\ unlikely to strongly boost approval numbers.
}

ecent weeks have witnessed two US legislative initiatives that
promise to not only extend the range of conditions for which a
provisional drug approval could be obtained but also accelerate patient
access to medications that address unmet needs. The Transforming
the Regulatory Environment to Accelerate Access to Treatments
(TREAT) and the Faster Access to Specialized Treatments Act (FAST),
championed by the Biotechnology Industry Organization (BIO) and
patient advocacy groups, codify the precept that any medicine tar-
geted to a condition with no effective treatment should qualify for
'accelerated approval' The proposed legislation is intended to galva-
nize the US Food and Drug Administration (FDA) to adopt a broader
definition of what qualifies for expedited approval and provide greater
consistency and clarity in its decision making. But implementation of
an expanded accelerated approval program will be a major challenge.
The legislation provides no additional funding to the FDA. And for
most diseases, the science behind intermediate clinical and surrogate
markers simply remains too preliminary.
Drug makers can currently use three distinct mechanisms to accelerate market entry of a new therapy. 'Fast Track' status offers frequent access to FDA for discussion of trial design and requisite supporting data (typically it is granted during phase 2 trials); 'Priority Review' is for drugs that address unmet needs and/or offer major advances over existing treatments, reducing the review time to 6 months (from 10 months); and 'Accelerated Approval', a mechanism instituted by FDA in 1992 at the behest of AIDS activists, uses surrogate endpoints to replace longer-term clinical outcomes (e.g., overall survival or symptom improvement) in approval. Approvals based on surrogate endpoints require post-marketing trials to verify the anticipated clinical benefit.

To date, the FDA has applied accelerated approvals predominantly in cancer and AIDS, where surrogate markers that correspond to broader clinical outcomes (e.g., overall survival) have been established. But the pathway hasn't exactly been a mother lode of new approvals. In 2011, only 3 of 30 new molecular entities went through accelerated approval. Overall, in two decades, only four drugs a year have emerged from this pathway.

It is this underwhelming output that the new legislation seeks to address. Both the TREAT and FAST bills explicitly state that accelerated approval should be applied to a broad range of "serious or life-threatening diseases." In addition, they reiterate that in addition to surrogate endpoints, intermediate clinical endpoints before "irreversible morbidity or mortality" (e.g., absence of residual cancer or decrease in necrosis/inflammation score) should be adopted by FDA.

It seems unlikely, however, that legislation will have a major influence on approval success and speed. First, although cancer and HIV dominate current accelerated approvals, other indications account for around $25 \%$ of them. The simple act of codifying a broader indication definition seems hardly likely to lead to a sea change in how FDA or sponsors treat the pathway.

Second, the Accelerated Approval pathway is not without uncertainties. In recent years, FDA has been ratcheting up the requirements. Much of the controversy has surrounded Richard Pazdur, the director of the Center for Drug Evaluation and Research (CDER)'s Office of Hematology Oncology Products, who has expressed skepticism about the use of single-arm, 'quick' trials. The oncology experience doesn't augur well for indications less traveled.

Which leaves perhaps the biggest question of all: can appropriate surrogate endpoints be defined? Neither TREAT nor FAST define what endpoints would work. The problem for FDA is thus not whether the agency accepts worked-up surrogate or clinical endpoints. The problem is that in many cases these endpoints simply don't exist.

It usually takes years to generate scientific consensus around a qualified marker-something that would be particularly difficult in rare diseases where few researchers are working. Paradoxically, rare diseases in which disease progression is slow and definitive proof of efficacy requires prolonged monitoring of patients are just the indications that stand to benefit most from biomarker research.

One way to generate such data will be consortia like Critical Path and the Innovative Medicines Initiative or research at the FDA itself. In recent Congressional testimony, CDER director Janet Woodcock said that FDA plans to release a draft guidance on the pathologic complete response (the absence of residual cancer) as a surrogate endpoint in high-risk breast cancers sometime soon.

But these cases are a drop in the ocean when one considers how few published biomarker studies are adequately powered and well documented, avoid sampling bias and accurately estimate error. According to Woodcock, as much as $75 \%$ of published biomarker associations in the literature are not replicable.

Thus, an optimistic view of this legislation is that it builds on a tried and tested framework. And despite initial reservations about surrogate markers, Accelerated Approval has worked for AIDS and oncology. Back then, the science was imperfect and yet the program still brought drugs to patients. If TREAT and FAST spur FDA to further clarify regulatory requirements for this pathway, the quality of phase 2 trial design will likely be improved and attrition will likely decrease. They may even allow some companies to reach the market and generate revenue earlier while spending less on R\&D.

But this is not a case of biomarker science being 'imperfect'. For many indications, the science has barely been started. And without a massive investment in biomarker research, it's hard to see how the FDA will be able to make rapid decisions for drugs. Until the science catches up, this legislation looks more like an evolution than a revolution in drug approval. 